\title{
Für- und Selbstsorge
}

Liebe Leserinnen, liebe Leser

Manchmal beginnen Menschen, sich selbst zu vernachlässigen: Die einen putzen sich ihre Zähne nicht mehr, hören auf die Kleider zu wechseln, vergessen ihre Medikamente, trinken zu viel Alkohol oder bewegen sich kaum. Andere gönnen sich keine Pause, treffen ihre Freunde nicht mehr, können sich nicht von kaputten Dingen trennen, leben in einer schmutzigen oder vermüllten Wohnung.

Dass es jemand nicht mehr schafft, sich um seine körperliche und geistige Gesundheit zu sorgen, kann viele Gründe haben: Trauer um einen geliebten Menschen, Einsamkeit, Sucht, finanzielle Probleme, körperliche Einschränkungen, Schmerzen, Depressionen, Angst, fehlende Anerkennung von anderen, wenig Wertschätzung sich selbst gegenüber. Manchmal vernachlässigt sich jemand aber auch ohne ersichtlichen Grund.

Menschen, die nicht auf ein Minimum an Hygiene und nicht auf ihre Gesundheit achtgeben können, sind für Pflegepersonen eine grosse Herausforderung. Insbesondere ambulante Pfleger_innen haben oft mit Menschen zu tun, die sich selbst vernachlässigen, und sie ekeln sich vielleicht vor ihnen bzw. deren verschmutzter Wohnung nicht immer kann eine professionelle Haltung verhindern, dass ein Lebensstil verurteilt wird, der von den eigenen (bürgerlichen) Wertvorstellungen abweicht.

Mit dem Aufeinanderprallen unterschiedlicher Lebensweisen und Werte setzt sich Esther Indermaur in ihrem Beitrag auseinander. Sie beschreibt, welchen Arten von Selbstvernachlässigung Spitex-Mitarbeitende begegnen können und wann bzw. wie eine Intervention sinnvoll ist. Melanie Werren und Daniela Händler-Schuster zeigen ebenfalls, wie herausfordernd die Pflege von Menschen ist, die sich selbst vernachlässigen, und erklären, warum Wertschätzung in diesen Settings unheimlich wichtig ist. Anna Gogl plädiert im Gespräch mit Sabine Hahn für kreatives, pflegerisches Handeln jenseits bürgerlicher Normen und Siegfried Huhn schreibt über das Messie-Syndrom, bei dem es sich um viel mehr handelt als die blosse Unfähigkeit Ordnung zu halten.

Evelyn Kraft nähert sich dem Thema von der anderen Seite und zeigt, warum Selbstfürsorge (für Pflegende) (jetzt gerade) so wichtig ist, und gibt Tipps für körperliche,

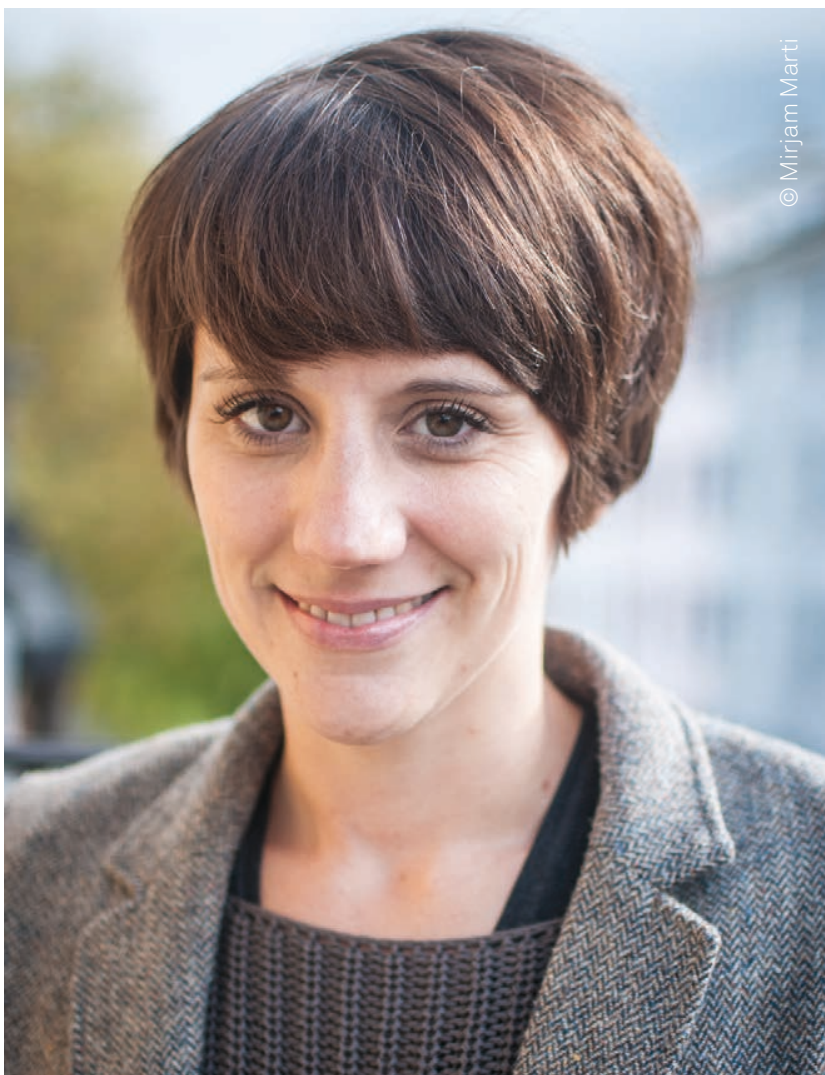

emotionale, kognitive, soziale und spirituelle Selbstfürsorge. Auch Thomas Holtbernd ist überzeugt, dass Fürsorge eng an Selbstsorge gebunden ist und lotet dieses Verhältnis aus, während sich Lea Frei dem Thema Selbstpflege bei Krankheit annimmt, indem sie eine Frau porträtiert, die während einer Chemotherapie ihre Haare verliert.

In der ersten NOVAcura dieses Jahres erwarten Sie ausserdem wie immer die Beiträge der übrigen Rubriken, speziell in dieser Ausgabe der Ausblick auf die SchwerpunktThemen des kommenden Jahres und der erste Beitrag der Reihe „Geschlechtlichkeit bei Menschen mit geistiger Behinderung" von Lotte Habermann-Horstmeier.

Ich wünsche Ihnen eine spannende Lektüre und grüsse Sie herzlich.

Eveline Kühni 


\section{Inhalt}

Editorial

Für- und Selbstsorge

Eveline Kühni

Schwerpunkt

Wenn Fingernägel schmutzig, die Wohnung chaotisch und Beziehungen

9 abgebrochen werden ...

Melanie Werren \& Daniela Händler-Schuster

Autonomie und Selbstvernachlässigung

Siegfried Huhn

Wenn das Selbst verwahrlost und zur existenziellen Provokation

für andere wird

Thomas Holtbernd

Kamingespräch

Sabine Hahn

Selfcare für Pflegefachpersonen

Evelyn Kraft

Gut betucht

Lea Frei

Medientipps zum Thema

Lena-Marie Wimmel

\begin{tabular}{|c|c|c|}
\hline \multirow[t]{2}{*}{ Folkus } & $\begin{array}{l}\text { Von der schwierigen Kunst der Abhängigkeit } \\
\text { Heinz Rüegger }\end{array}$ & 35 \\
\hline & $\begin{array}{l}\text { "Ja, die vermisst mich halt. Die hat mich halt total lieb.“ } \\
\text { Lotte Habermann-Horstmeier }\end{array}$ & 39 \\
\hline \multirow[t]{2}{*}{ Palliative Care } & $\begin{array}{l}\text { PACE: Palliative Care ergänzender Begleitdienst } \\
\text { Matthias Staub \& Elisabeth Jordi }\end{array}$ & 44 \\
\hline & $\begin{array}{l}\text { Flüssigkeitssubstitution in der letzten Lebensphase - } \\
\text { eine Frage der Perspektive? } \\
\text { Imane Henni Rached }\end{array}$ & 47 \\
\hline Pflegen zu Hause & $\begin{array}{l}\text { Selbstvernachlässigung } \\
\text { Esther Indermaur }\end{array}$ & 51 \\
\hline Bildung & $\begin{array}{l}\text { Pflege und Forschung } \\
\text { Brigitte Teigeler }\end{array}$ & 54 \\
\hline \multirow[t]{3}{*}{ Dementia Care } & $\begin{array}{l}\text { Mehr Lebensqualität, weniger Psychopharmaka } \\
\text { Brigitte Teigeler }\end{array}$ & 56 \\
\hline & $\begin{array}{l}\text { "Gemeinsam werden wir es schaffen ...“ } \\
\text { Veronika Müller \& Angela Schnelli }\end{array}$ & 60 \\
\hline & $\begin{array}{l}\text { Reicher Erfahrungsschatz einer Demenz-Fachfrau } \\
\text { Buchtipp }\end{array}$ & 64 \\
\hline à propos & & 65 \\
\hline Vorschau/Termine & & 66 \\
\hline
\end{tabular}

